\title{
Comparison of the Influences of Surface Texture and Boundary Slip on Tribological Performances
}

\author{
Qiyin Lin ${ }^{1,2}$ and Baotong $\mathrm{Li}^{2}$ \\ ${ }^{1}$ Fuli School of Food Equipment Engineering and Science, Xi'an Jiaotong University, Xian, Shaanxi 710049, China \\ ${ }^{2}$ State Key Laboratory for Manufacturing Systems Engineering, Xian Jiaotong University, Xian, Shaanxi 710049, China \\ Correspondence should be addressed to Baotong Li; baotong.me@mail.xjtu.edu.cn
}

Received 17 May 2015; Revised 15 July 2015; Accepted 15 July 2015

Academic Editor: Rama S. R. Gorla

Copyright (c) 2015 Q. Lin and B. Li. This is an open access article distributed under the Creative Commons Attribution License, which permits unrestricted use, distribution, and reproduction in any medium, provided the original work is properly cited.

\begin{abstract}
Close attentions have been widely paid to the engineering textured and slip surfaces for improving bearing tribological performances. Comparison studies on the tribological characteristics of slip and textured surfaces are carried out in this work. The analysis results point out that the influences of surface texture and boundary slip on tribological performances of slider bearing are strongly similar. For the determinate surface textures, there is one and only value of slip velocity to make the tribological performances of textured and slip surfaces in agreement. The corresponding relation between the slip velocity and the texture structure parameters is also obtained, and the size of slip velocity is directly related to the texture geometry parameters including its position parameters. This study will help us to further understand the relationship between boundary slip and surface texture and also the slip phenomenon.
\end{abstract}

\section{Introduction}

In order to enhance bearing performance, some innovative approaches are developed, for instance, engineering textured and slip bearing, as well as novel-configuration bearing [1-6] . Surface texture is growing, being used for different purposes such as the improvement of tribological performances. The increase in the load-carrying capacity under hydrodynamic lubrication is one of the most successful applications of surface texture in tribological field, and many attentions have been paid to it.

As early as 1950, Salama experimentally and theoretically investigated the influence of macroroughness (can be regarded as surface texture) on the performance of thrust bearing and indicated that it owns two functions: feeding lubricant and generating hydrodynamic film [7]. Hamilton et al. pointed out that the improvement of loadcarrying capacity resulted from the asymmetric pressure distribution induced by surface texture [8]. Due to the cavitation at the front-end of dimple, the negative pressure is prevented and the asymmetric pressure distribution is obtained. The high fluid pressure at the back-end of dimple (convergence zone) counteracts the low fluid pressure at the front-end of dimple (divergence zone), finally resulting in the enhancement of load-carrying capacity. Anno et al. found out that microasperity acted like a microhydrodynamic bearing and generated load support [9]. Tønder indicated that texturing (roughening) the inlet region of plane pad could obtain hydrodynamic action and positive lift, and the inlet roughness/texture produced an equivalent virtual effect of a Rayleigh step [10]. Meanwhile, microgeometries in inlet zone make the flow into the bearing meet less resistance than it out of the bearing; thus, the available lubricant within the pressure-generating zone is increased. Brizmer et al. pointed out that two concepts could be used to produce load-carrying capacity in parallel sliding bearing: a collective dimples effect in partial textured surface and an individual dimple effect in full textured surface [11]. For partial textured surface, each dimple strongly affects its neighboring dimples, resulting in a collective effect of the dimples and a steplike pressure distribution over the textured zone. For full textured surface, the dimples do not interact (individual effect), resulting in a periodic pressure distribution. An inlet suction mechanism was demonstrated by Olver et al. [12]. 
The pressure reduction from the inlet to the front-end of dimple/pocket results in suction of lubricant into bearing and thus enhances load support. This inlet suction mechanism makes it possible for parallel or low convergence bearing to generate a lubricant film and support an imposed load. Arghir et al. were enlightened that the pressure generation as well as load support within surface texture is because of the convective-inertia effect [1]. The transient behavior of textured bearing got the attention of Gherca et al. and they indicated that the load support produced by moving textured surface was based on the squeeze effect of the leading edge of surface texture [13]. Another mechanism named as balancing wedge action was proposed by Yagi and Sugimura [14]. This mechanism suggests that surface texture on one of the mating surfaces increases the convergence ratio between the surfaces, resulting in the promotion of the whole wedge action in the contact area.

Much more studies focus on the influences of various texture geometries such as shape, depth, and aspect-ratio on bearing performances. The effects of texture geometry such as texture depth, width, number, and location of texture on slider bearing performances were studied by Fowell et al. [15]. In order to discuss optimal texturing properties for minimizing friction or maximizing fluid film thickness, a study on the influence of different texturing parameters on hydrodynamic performance was proposed by Dobrica et al. [16]. Fesanghary and his coworker paid many attentions on the optimization design of texture shape and geometry for improving load-carrying capacity based on sequential quadratic programming algorithm [5]. The optimum texture to gain maximum load-carrying capacity is chevrontype shape with flat front for unidirectional sliding, and it consists of pairs of the trapezoid-like shapes for bidirectional sliding. The effects of the parameters of orientation ellipse dimple such as area density and textured fraction on the performances of hydrodynamic lubrication were analyzed by Ji et al. [17]. Besides, Qiu et al. investigated the influence of such texture parameters as shape and density on the friction coefficient and stiffness of gas-lubricated bearing, and the optimal geometry in terms of the maximum load-carrying capacity was also obtained [18]. Hao et al. pointed out that surface texture with lower area density and larger size had a better lubrication performance [19].

Textured surface may be able to induce boundary slip when liquid flows on it, which means that liquid molecules do not adhere to solid surface and there is a relative velocity at fluid-solid interface, and such textured surfaces are commonly known as superhydrophobic surfaces [2024]. Superhydrophobic surface can be fabricated from the modification of surface microtopography (e.g., surface texture) and surface energy. Researchers make a lot of work to study the influences of slip surface on bearing tribological performances. A review on the hydrodynamic lubrication with slip surface was presented recently by Zhang [25]. Spikes developed a hydrodynamic lubrication model based on an extended Reynolds equation and analyzed the application of slip surface [26]. He showed that slider bearing with slip against its stationary surface could generate both an increase in load support and a reduction in friction. Both the slider bearing and hydrodynamic journal bearing with a heterogeneous slip/noslip surface were analyzed by Salant and Fortier; the results demonstrated that boundary slip would result in good load-carrying capacity and low friction $[2,27]$. Chen et al. demonstrated that the boundary slip condition would move more fluid at one cross section than at a cross section without slip [28]. The study of Wu indicated that the enhanced effect of slip surface on hydrodynamic journal bearing performance was much greater at a small eccentricity ratio than at a large eccentricity ratio [29]. The location and area of slip surface will significantly affect the load-carrying capacity of both the slider bearing and journal bearing $[30,31]$. Aurelian et al. compared the effect of wall slip condition with the effect of surface texture under similar lubrication condition and pointed out that the tribological behavior of slip surface and textured surface was similar [32]. Furthermore, the influences of boundary slip on heat generation were noticed by Mahmoud and Waheed [3]. Sharma et al. also pointed out that velocity slip would reduce heat transfer rate [33].

Above all, most studies focus on the individual influence of surface texture or boundary slip on tribological performances of slider and journal bearings, respectively, and a little other literatures pay attention to the synthesized impacts of surface texture and boundary slip. There are many important discoveries demonstrated by these published researches, but there are also limitations: although some results indicate that textured surface and slip surfaces have similar tribological characteristic, the comparison and analysis on their connection are still insufficient; for example, the relationship between slip velocity and texture parameters is less underlined. A comparison analysis on the effects of slip and textured surfaces on tribological performances of slider bearing is performed in this study, and a corresponding relation between the slip velocity and texture parameters is also deduced. Some interesting results are obtained, which will help people to further understand surface texture and boundary slip.

\section{Theory and Model}

For continuous isoviscous incompressible fluid medium, the continuity and Navier-Stokes equations are used to predict the performances of the fluid domain, which are expressed as follows:

$$
\begin{aligned}
& \frac{\partial u}{\partial x}+\frac{\partial v}{\partial y}+\frac{\partial w}{\partial z}=0 \\
& \rho\left(u \frac{\partial u}{\partial x}+v \frac{\partial u}{\partial y}+w \frac{\partial u}{\partial z}\right) \\
& =-\frac{\partial p}{\partial x}+\mu\left(\frac{\partial^{2} u}{\partial x^{2}}+\frac{\partial^{2} u}{\partial y^{2}}+\frac{\partial^{2} u}{\partial z^{2}}\right), \\
& \rho\left(u \frac{\partial v}{\partial x}+v \frac{\partial v}{\partial y}+w \frac{\partial v}{\partial z}\right) \\
& =-\frac{\partial p}{\partial y}+\mu\left(\frac{\partial^{2} v}{\partial x^{2}}+\frac{\partial^{2} v}{\partial y^{2}}+\frac{\partial^{2} v}{\partial z^{2}}\right)
\end{aligned}
$$




$$
\begin{aligned}
& \rho\left(u \frac{\partial w}{\partial x}+v \frac{\partial w}{\partial y}+w \frac{\partial w}{\partial z}\right) \\
& =-\frac{\partial p}{\partial z}+\mu\left(\frac{\partial^{2} w}{\partial x^{2}}+\frac{\partial^{2} w}{\partial y^{2}}+\frac{\partial^{2} w}{\partial z^{2}}\right),
\end{aligned}
$$

where $u / v / w$ are velocity components, $x / y / z$ are Cartesian coordinates, $\rho$ is fluid density, $p$ is fluid pressure, and $\mu$ is viscosity.

A published slider bearing with square textures (dimples) demonstrated by Marian et al. in [34] is used to investigate and compare the influences of surface texture and boundary slip on tribological performances. The schematic diagram of this slider bearing is presented in Figure 1, and its physical photograph was shown in Figure 9 of [34]. The inner radius of this slider bearing $r_{i}$ is $28.5 \mathrm{~mm}$ and its outer radius $r_{o}$ is $45 \mathrm{~mm}$. The width of oil supply channel at mean radius is $2 \mathrm{~mm}$. This slider bearing consists of 12 bearing pads. The dimple width is $200 \mu \mathrm{m}$ and its depth is $9 \mu \mathrm{m}$. The area density of dimples, that is, the ratio of total area of the dimples to total area of the textured surface, is 0.25 ; that is, $\left(l_{d} / l_{c}\right)^{2}=0.25$. The textured fraction is 0.5 in circumferential direction and is 0.9 in radial direction; that is, $\theta_{t} / \theta_{p}=0.5$ and $B_{t} / B_{p}=0.9$. Thus, dimple number is 22 in circumferential direction and is 38 in radial direction.

The corresponding slider bearing with slip surface is presented in Figure 2. The only difference compared to the textured bearing as shown in Figure 1 is in these regions where dimples locate: these regions are flat in the slip bearing and slip boundary condition is applied onto them. Both the other stationary wall and moving wall are applied with traditional noslip boundary condition. Due to the periodicity of the bearing geometry, only a single bearing pad is built up for analysis in this study and the tribological performance of the whole slider bearing is obtained from multiplying the corresponding performance of the single bearing pad by 12 .

In the experiments conducted by Marian et al., the rotational speeds $\omega$ of moving wall were from $500 \mathrm{rpm}$ to $800 \mathrm{rpm}$, and the imposed external axial loads $W$ were $100 \mathrm{~N}$ and $200 \mathrm{~N}$ [34]. It should be pointed out that, in order not to cumulate the error between the experimental and the theoretical values of the fluid film thickness $h_{c}$, their values in these slip slider bearings of this work are set equal to the experimental values presented in [34] under the same rotational speed. The experimental values of fluid film thickness under different working conditions are presented in Table 1 again for convenience. And the axial loads are the calculated values in this theoretical study, but they were input data in the experimental test of [34]. The other conditions in this study keep the same as the experimental conditions in [34]. Both the inlet and outlet pressure are equal to the ambient pressure. The density of lubricant is $848 \mathrm{Kg} \cdot \mathrm{m}^{-3}$ and its viscosity $\mu$ is $0.022 \mathrm{~Pa} \cdot \mathrm{s}$.

To the geometry structure of the slider bearing in this study, only the first dimple column exists in small negative pressure, and the cavitation phenomena can be neglected due to the fact that the area of the first dimple column is insignificant compared to the total area of the bearing, and this is
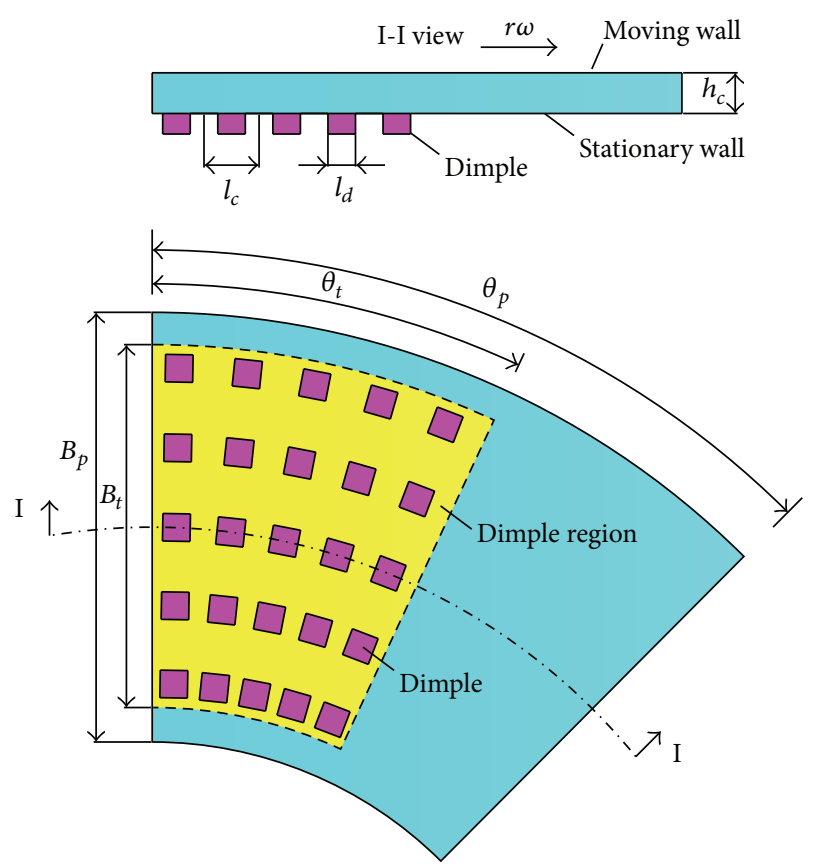

FIGURE 1: Schematic diagram of a bearing pad of the slider bearing with square dimples.

TABLE 1: Fluid film thickness $h_{c}$.

\begin{tabular}{lcc}
\hline \multicolumn{1}{l}{$h_{c}$} & $100 \mathrm{~N}$ & $W$ \\
\hline$\omega$ & & $200 \mathrm{~N}$ \\
$500 \mathrm{rpm}$ & $17.08 \mu \mathrm{m}$ & \\
$600 \mathrm{rpm}$ & $18.13 \mu \mathrm{m}$ & $12.92 \mu \mathrm{m}$ \\
$700 \mathrm{rpm}$ & $18.75 \mu \mathrm{m}$ & $13.75 \mu \mathrm{m}$ \\
$800 \mathrm{rpm}$ & $19.17 \mu \mathrm{m}$ & $14.58 \mu \mathrm{m}$ \\
\hline
\end{tabular}

also confirmed by Marian et al. [34]. The result based on a testing analysis in [34] showed that the difference between the pressure considering cavitation and that without cavitation phenomena is extremely small; that is, the introduction of cavitation phenomena had negligible effect on the results, so the final analyses did not take the cavitation phenomena into account in [34]. Thus, the cavitation is not considered as well in this study, and the thermal effect is also neglected for decreasing computing time.

For noslip boundary condition, it is assumed that the liquid molecules adhere to solid surface strongly at the fluidsolid interface, so there is not relative velocity at fluid-solid interface; for slip boundary condition, it is assumed that the relative velocity between liquid and solid at the fluidsolid interface is not equal to 0 , and this relative velocity is defined as slip velocity $U_{s}$, as shown in Figure 3. There are two main slip boundary conditions: the Navier boundary condition and the limiting shear stress boundary condition $[2,26,27,29,31,32]$. The Navier boundary condition, also called as slip length boundary condition, states that the slip velocity $U_{s}$ is proportional to the surface shear rate $(\partial u / \partial y)$ and slip length $b$; that is, $U_{s}=b \cdot(\partial u / \partial y)$. The slip length $b$ is 

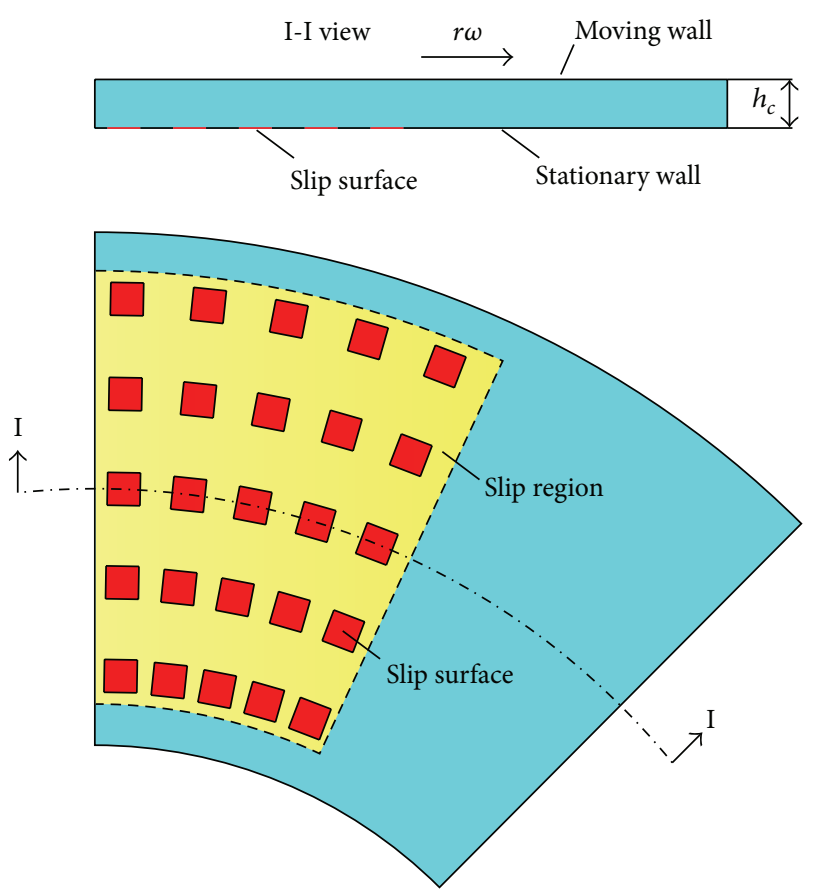

Figure 2: Schematic diagram of a bearing pad of the slider bearing with slip surfaces.

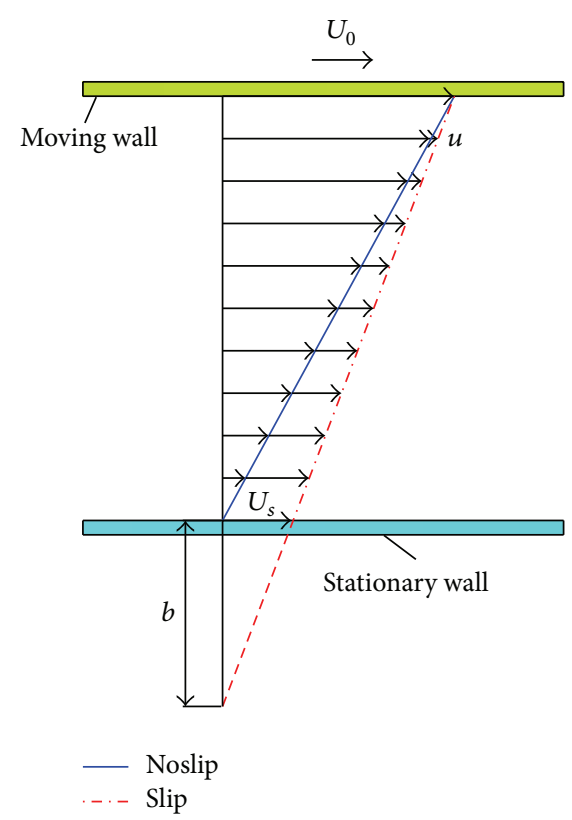

FIGURE 3: Comparison of the noslip and slip boundary phenomenon.

defined as the fictive distance below the solid surface, where the velocity extrapolates linearly to zero, as shown in Figure 3. The limiting shear stress boundary condition assumes that there is a limiting/critical shear stress $\tau_{c}$ at the fluid-solid interface and the wall slip occurs only when the wall shear stress $\tau_{0}$ reaches the critical value. If slippage occurs, the surface shear stress $\tau_{s}$ is equal to the critical value; that is, $\tau_{s}=\tau_{c}$ (if $\tau_{0} \geq \tau_{c}$ ), and $\tau_{s}=\tau_{0}$ (if $\tau_{0}<\tau_{c}$ ), where $\tau_{0}=\mu \cdot(\partial u / \partial y)$. However, the exact value of the slip length is difficult to determine for a certain structure, and the same problem still exists for critical shear stress.

Not only the Navier boundary condition but also the limiting shear stress boundary condition indicates that the slip velocity $U_{s}$ is always related to fluid velocity $U_{i}$ at the nearest region close to this fluid-solid interface. For the convenience, to determine the slip intensity, a slip-intensity factor $f$ is used; thus, a slip boundary condition with a slipintensity factor can be derived from the limiting shear stress boundary condition then and is expressed as

$$
U_{s}=f \cdot\left(U_{i}-n \cdot\left\langle U_{i} \cdot n\right\rangle\right) \text {, }
$$

where $n$ is the surface normal vector and this $\operatorname{part} n \cdot\left\langle U_{i} \cdot n\right\rangle$ represents the velocity component of $U_{i}$ in the surface normal direction. Equation (2) indicates that boundary slip occurs in the tangential direction, and the normal component of velocity is zero. Assume that the three components of slip velocity $U_{s}$ are $\left(u_{s}, v_{s}, w_{s}\right)$ and the three components of fluid velocity $U_{i}$ are $\left(u_{i}, v_{i}, w_{i}\right)$ under this coordinate system $x_{n^{-}} y_{n^{-}}$ $z_{n}$, where the direction of surface normal vector $n$ is $-z_{n}$, as shown in Figure 4. When (2) is applied, we have

$$
\begin{aligned}
& u_{s}=f \cdot u_{i}, \\
& v_{s}=f \cdot v_{i}, \\
& w_{s}=0 .
\end{aligned}
$$

For possessing physical meaning, the slip-intensity factor is restricted from 0 to 1 ; that is, $f \in[0,1]$. This condition $f=1$ represents a shear-free condition, that is, a perfect slip condition, and at this situation we have $\partial u / \partial y=0$; thus, this condition is also equivalent to the limiting shear stress slip boundary condition when the critical shear stress is zero. And this situation $f=0$ is equivalent to the stationary wall without slip, that is, noslip boundary condition with zero speed. This slip boundary condition with a slip-intensity factor is applied onto these slip surfaces in the following studies of this work. The numerical analyses are performed using OpenFOAM.

A careful check on mesh independence is conducted firstly to ensure the accuracy of numerical solution. The mesh independence analyses are based on this slip bearing model with $f=1$, when the rotational speed is $500 \mathrm{rpm}$ and the fluid film thickness is $17.08 \mu \mathrm{m}$ (i.e., the imposed external axial load in experiment was $100 \mathrm{~N}$ ); the analysis results indicate that the mesh density of 546 kilo is good enough for the accuracy of numerical solution; thus, the same mesh density is used for all the numerical models in this work. The tribological performances of the slip slider bearing predicted theoretically in this study are compared with the corresponding experimental and theoretical values of the textured slider bearing demonstrated in [34].

\section{Results}

The effects of slip-intensity factor $f$ on the load-carrying capacity and friction torque are illustrated in Figures 5 and 6. 


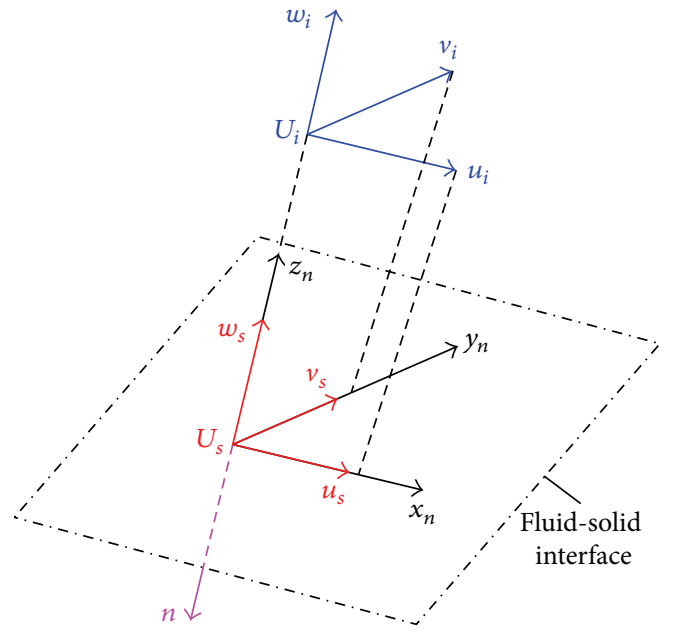

FIGURE 4: Schematic diagram of velocity.

TABLE 2: The values of slip-intensity factor $f$ when $W \approx 100 \mathrm{~N}$ and $200 \mathrm{~N}$.

\begin{tabular}{lcc}
\hline \multicolumn{1}{l}{$f$} & $W$ & \\
\hline$\omega$ & $100 \mathrm{~N} \pm 1 \%$ & $200 \mathrm{~N} \pm 1 \%$ \\
$500 \mathrm{rpm}$ & 0.775 & \\
$600 \mathrm{rpm}$ & 0.755 & 0.79 \\
$700 \mathrm{rpm}$ & 0.732 & 0.768 \\
$800 \mathrm{rpm}$ & 0.705 & 0.758 \\
\end{tabular}

For the textured bearing models corresponding to the slip bearing models whose results are shown in Figure 5, the imposed load-carrying capacity $W$ in Marian's experiment is $100 \mathrm{~N}$; and it is $200 \mathrm{~N}$ for that corresponding to the slip bearing models whose results are shown in Figure 6. The theoretical results demonstrate that the effects of slip-intensity factor on load-carrying capacity and friction torque are both monotone. The load-carrying capacity monotonically increases with the slip-intensity factor and the friction torque monotonically decreases with the slip-intensity factor. On the other hand, there is one and only value of slip-intensity factor $f$ which makes the predicted theoretical load-carrying capacity based on the slip boundary condition presenting in (2) equal to the imposed value in the experiment conducted by Marian et al.; and the corresponding values of this slipintensity factor $f$ are presented in Table 2, when the predicted theoretical load-carrying capacity is equal to the experiment value, that is, $100 \mathrm{~N}$ and $200 \mathrm{~N}$, respectively.

The theoretical friction torques based on these values of slip-intensity factor as shown in Table 2 are presented in Figure 7, and they are also compared to the measuring values and theoretical prediction values reported by Marian et al. [34]. The theoretical friction torques in this work and Marian's study are both smaller than the measured values reported in [34]. But the errors between the measured values and the theoretical values predicted in this study are smaller than that between the measured and theoretical values of [34]. The physical structure of dimples was really built up in the theoretical models of [34]. As pointed out by Marian et al., the differences between the measuring and theoretical friction torques may result from the measurement errors due to vibrations; the measuring values are oscillating over time and their mean values are used to make comparison with theoretical values.

The 2D dimensionless pressure distribution is illustrated in Figure 8 over a slip bearing pad. The dimensionless pressure $P$ is defined as $P=p h_{c}^{2} /(\mu r \omega L)$, where $L$ denotes the circumferential length of bearing pad. The fluid film thickness of this slip bearing is $10 \mu \mathrm{m}$, the numbers of slip surface in circumferential and radial directions are both 22, and other bearing parameters keep the same as the above. The slip-intensity factor $f$ is 0.8 .

The shape of this pressure distribution is similar to the pressure distribution of a step bearing, as shown in Figures 8 and 9 . There is a big pressure peak at the rear of the slip region. Figure 9(a) illustrates the 3D dimensionless pressure distribution over a bearing pad. The only difference compared to a step bearing is that there is an additional pressure peak (net pressure) at the rear of each slip surface. This can be seen much more clearly from Figure 9(b), which illustrates the pressure distribution over a section when $x / r_{i} \approx-1.29$. Each slip surface strongly affects its neighboring slip surface, and the pressure contributed by each slip surface has a collective effect. This pressure distribution based on slip bearing agrees well with that based on textured bearing as shown in Figure 6 of [34]. The dimple (texture) numbers in circumferential and radial directions are equal to the number of slip surface in these two directions, respectively. There is also a big pressure peak at the rear of the dimple region and an additional net pressure at the rear of each dimple as well as a collective effect on net pressure.

\section{Discussions}

The influences of slip surface on bearing tribological performances are very similar to those of concave texture (dimple) as demonstrated above. Each concave texture and slip surface will both induce a pressure peak (net pressure) locating at its rear. The global or cumulative impact of concave textures and slip surfaces will both result in a big pressure peak which just locates at the rear of texture region and slip region. For the same bearing models except these zones of texture and slip, the value of slip-intensity factor is one and only value to make the bearing tribological performances predicted by the slip boundary condition presenting in (2) equal to the tribological performances of the corresponding textured bearing.

The relationship between the slip velocity and the texture parameters will be deduced in the following, based on two 2D parallel slider bearing models with a texture and a slip surface in the stationary wall, respectively. These two slider bearing models are illustrated in Figure 10.

For the 2D parallel slider bearing model, the governing equations (1) can be simplified and reduced to

$$
\frac{\partial^{2} u}{\partial y^{2}}=\frac{1}{\mu} \cdot \frac{\partial p}{\partial x} .
$$




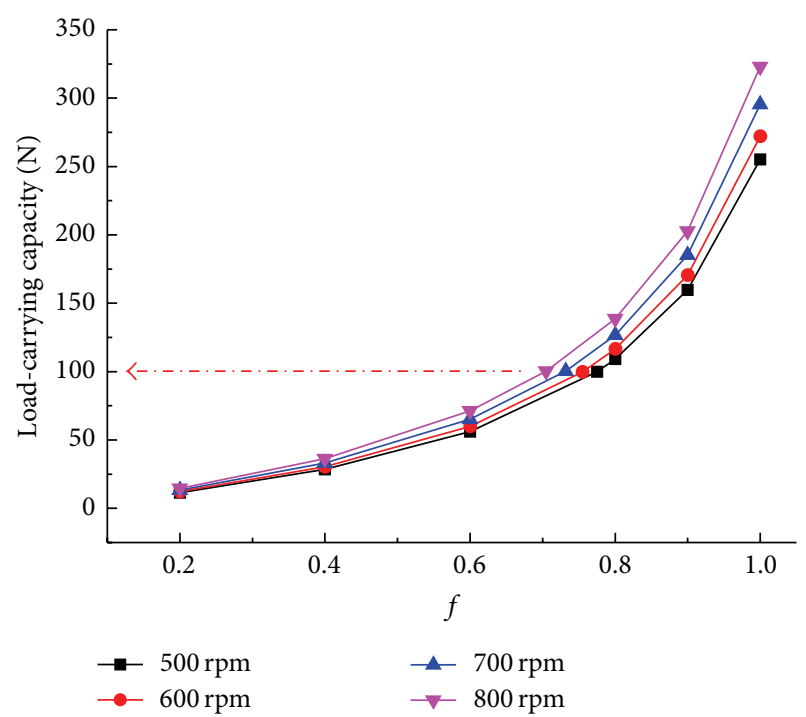

(a) Load-carrying capacity

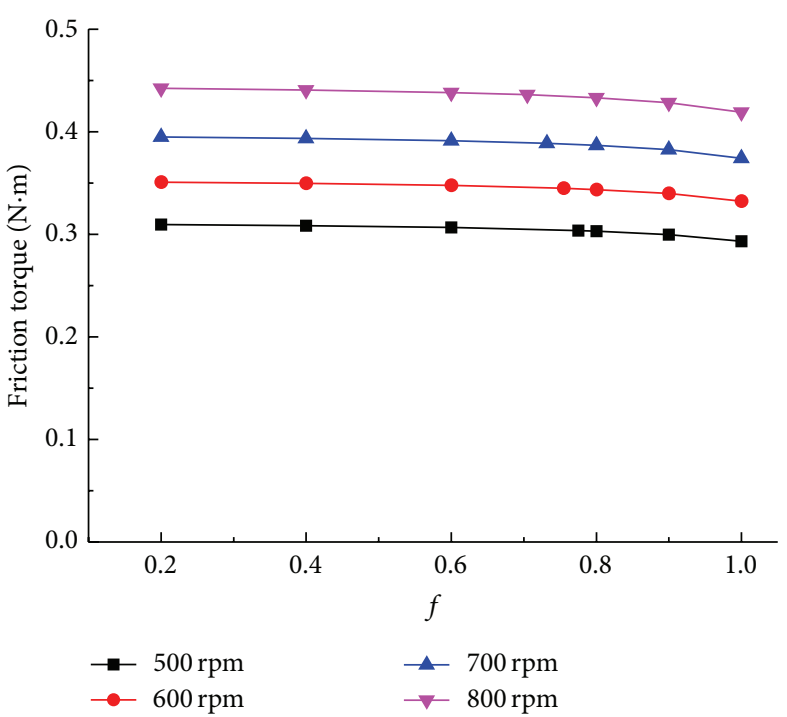

(b) Friction torque

FiguRE 5: Tribological performances versus slip-intensity factor based on these bearing models with $W=100$.

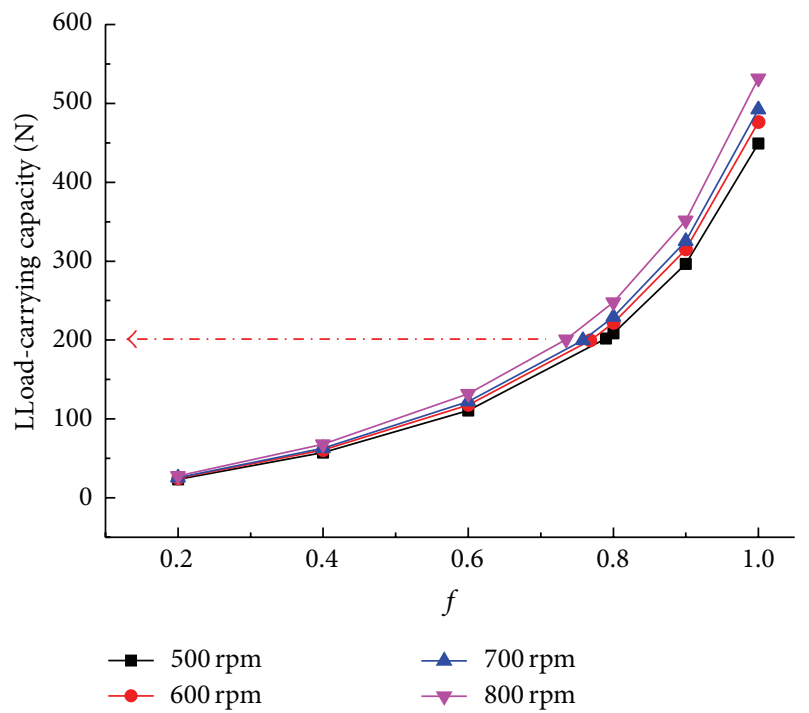

(a) Load-carrying capacity

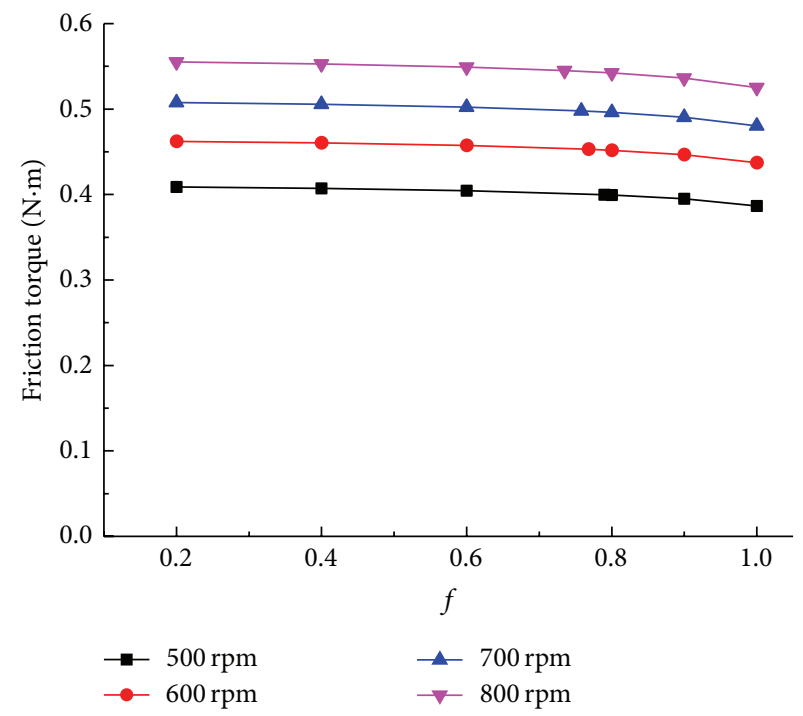

(b) Friction torque

FIGURE 6: Tribological performances versus slip-intensity factor based on these bearing models with $W=200$.

The speed of moving plate is $U_{0}$ and the slip velocity at the slip surface of stationary plate is $U_{s}$. The film thickness at dimple zone is $h_{d}$ and it is $h_{0}$ at the other noslip and slip zones. With the boundary conditions, $u(h)=U_{0}$ and $u(0)=0$ at noslip-surface zone or $u(0)=U_{s}$ at slip-surface zone; the fluid velocity at noslip-surface zone can be given as

$$
u_{\text {noslip }}=\frac{1}{2 \mu} \cdot \frac{\partial p}{\partial x} y^{2}+\left(\frac{U_{0}}{h}-\frac{h}{2 \mu} \cdot \frac{\partial p}{\partial x}\right) y
$$

And the fluid velocity at slip-surface zone is given as

$$
u_{\text {slip }}=\frac{1}{2 \mu} \cdot \frac{\partial p}{\partial x} y^{2}+\left(\frac{U_{0}}{h}-\frac{h}{2 \mu} \cdot \frac{\partial p}{\partial x}\right) y+\frac{h-y}{h} U_{s} \text {, }
$$

where $h$ represents the film thickness. The last term of the right hand side of (6) represents the contribution of boundary slip to fluid velocity. Thus, from the velocity equation, the flow rate $Q$ at a cross section can be obtained by the following:

$$
Q=\int_{0}^{h_{0}} u \cdot d y .
$$

So, the flow rates at noslip- and slip-surface zones are given, respectively, by

$$
\begin{aligned}
Q_{\text {noslip }} & =\frac{U_{0} h}{2}-\frac{h^{3}}{12 \mu} \cdot \frac{\partial p}{\partial x}, \\
Q_{\text {slip }} & =\frac{U_{0} h}{2}-\frac{h^{3}}{12 \mu} \cdot \frac{\partial p}{\partial x}+\frac{U_{s} h}{2} .
\end{aligned}
$$




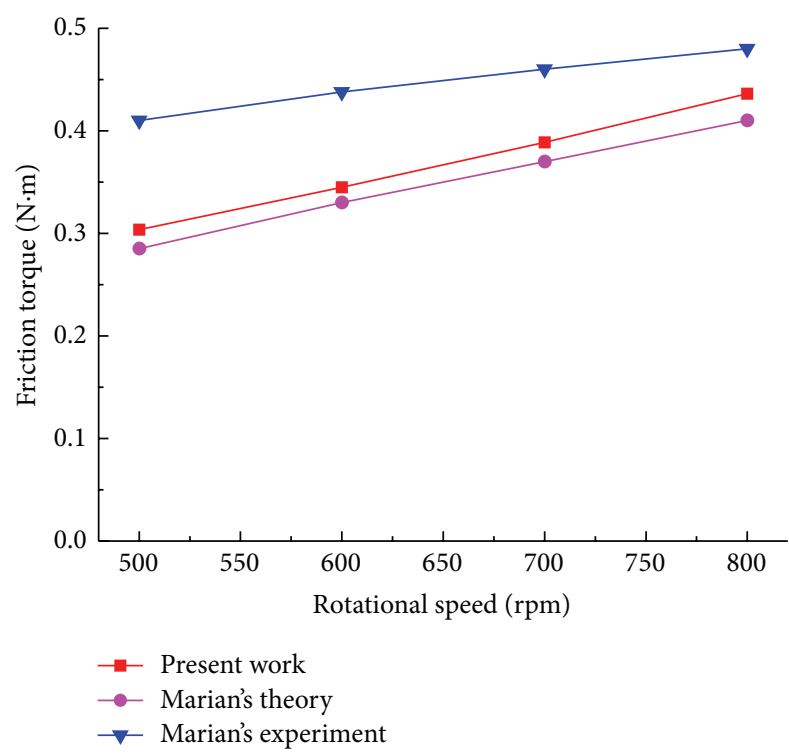

(a) When $W=100 \mathrm{~N}$

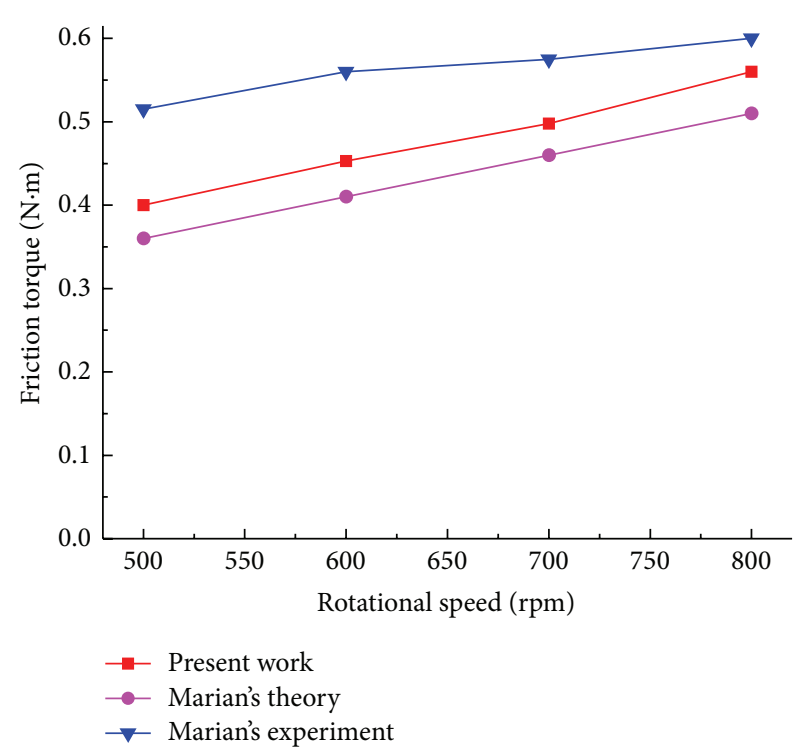

(b) When $W=200 \mathrm{~N}$

FIgURE 7: Comparison on friction torque.

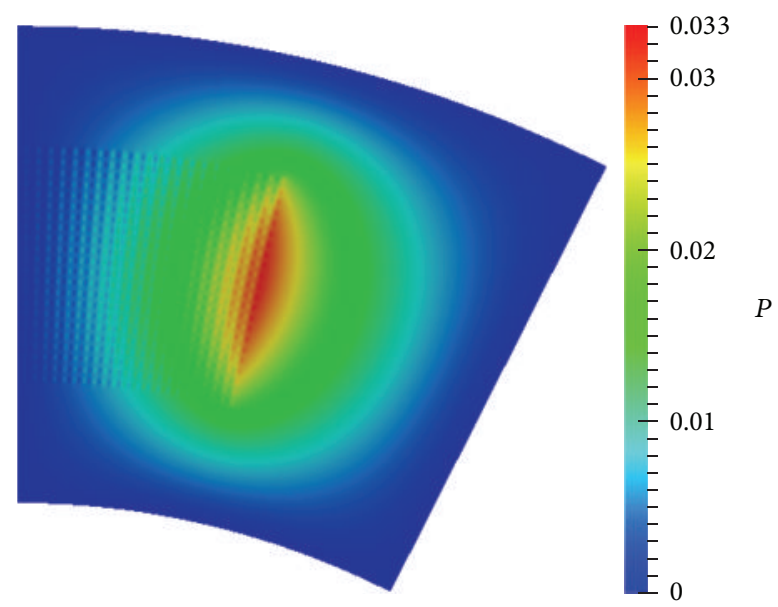

Figure 8: 2D pressure distribution over a bearing pad.

The last term of the right hand side of (9) represents the contribution of boundary slip to flow rate.

For these parallel slider bearings, the film thickness is constant, so this part $\partial p / \partial x$ is also constant; that is, the pressure variation is linear. For the slider bearing with a dimple, the flow rate for each region can thus be given by

$$
\begin{aligned}
& Q_{\mathrm{AB}}=\frac{U_{0} h_{0}}{2}-\frac{h_{0}^{3}}{12 \mu} \cdot\left(\frac{p_{2}-p_{1}}{a}\right), \\
& Q_{\mathrm{BC}}=\frac{U_{0} h_{d}}{2}-\frac{h_{d}^{3}}{12 \mu} \cdot\left(\frac{p_{3}-p_{2}}{b}\right), \\
& Q_{\mathrm{CD}}=\frac{U_{0} h_{0}}{2}-\frac{h_{0}^{3}}{12 \mu} \cdot\left(\frac{p_{4}-p_{3}}{c}\right) .
\end{aligned}
$$

The ambient pressure is applied onto the inlet and outlet; that is, $p_{1}=p_{4}=p_{\text {atm }}$. Because the flow is the continuity, the flow rate at every cross section is the equivalence; that is, $Q_{\mathrm{AB}}=Q_{\mathrm{BC}}=Q_{\mathrm{CD}}$. From $Q_{\mathrm{AB}}=Q_{\mathrm{CD}}$ and $Q_{\mathrm{BC}}=Q_{\mathrm{CD}}$, respectively, we have

$$
\begin{aligned}
& \frac{p_{2}-p_{\mathrm{atm}}}{a}=\frac{p_{\mathrm{atm}}-p_{3}}{c}, \\
& \frac{U_{0} h_{d}}{2}-\frac{h_{d}{ }^{3}}{12 \mu} \cdot\left(\frac{p_{3}-p_{2}}{b}\right) \\
& =\frac{U_{0} h_{0}}{2}-\frac{h_{0}{ }^{3}}{12 \mu} \cdot\left(\frac{p_{\mathrm{atm}}-p_{3}}{c}\right) .
\end{aligned}
$$

Combining (11) and (12) to eliminate $p_{2}$, we have

$$
p_{3}=p_{\mathrm{atm}}+\frac{6 \mu U_{0} b c\left(h_{d}-h_{0}\right)}{(a+c) h_{d}{ }^{3}+b h_{0}{ }^{3}} .
$$

Thus, combining (11) and (13), we then have

$$
p_{2}=p_{\mathrm{atm}}-\frac{6 \mu U_{0} a b\left(h_{d}-h_{0}\right)}{(a+c) h_{d}{ }^{3}+b h_{0}{ }^{3}} .
$$

For the slider bearing with a slip region, the flow rate for each region can thus be given by

$$
\begin{aligned}
& Q_{\mathrm{AB}}^{\prime}=\frac{U_{0} h_{0}}{2}-\frac{h_{0}{ }^{3}}{12 \mu} \cdot\left(\frac{p_{2}^{\prime}-p_{1}^{\prime}}{a}\right), \\
& Q_{\mathrm{BC}}^{\prime}=\frac{U_{0} h_{0}}{2}-\frac{h_{0}^{3}}{12 \mu} \cdot\left(\frac{p_{3}^{\prime}-p_{2}^{\prime}}{b}\right)+\frac{U_{s} h_{0}}{2}, \\
& Q_{\mathrm{CD}}^{\prime}=\frac{U_{0} h_{0}}{2}-\frac{h_{0}^{3}}{12 \mu} \cdot\left(\frac{p_{4}^{\prime}-p_{3}^{\prime}}{c}\right) .
\end{aligned}
$$




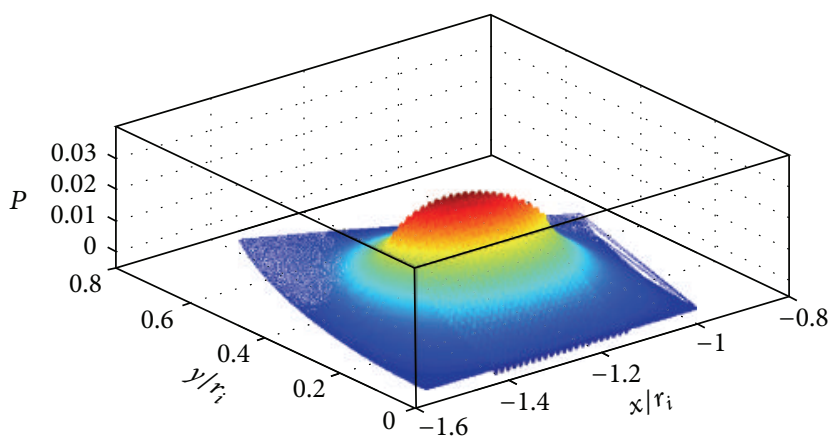

(a) Over a slip bearing pad

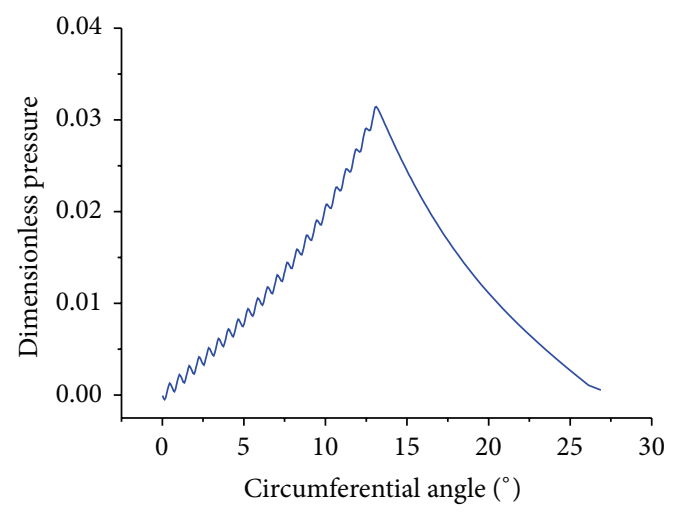

(b) At this section when $x / r_{i} \approx-1.29$

FIgUre 9: Pressure distribution.
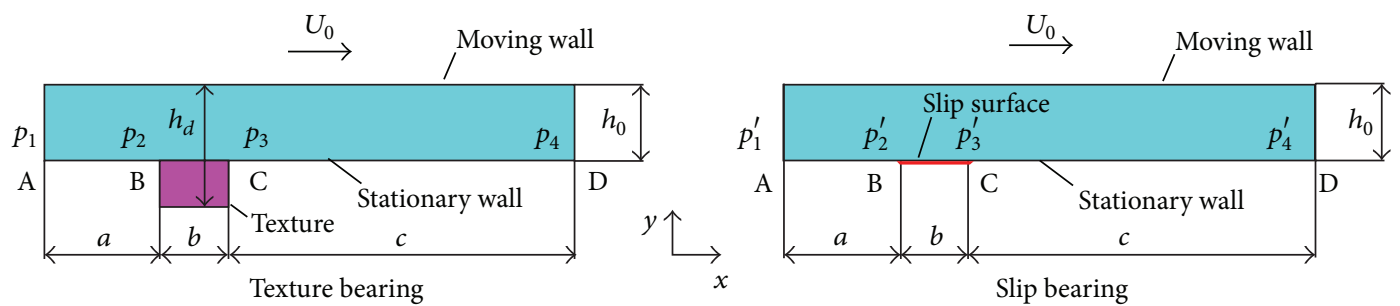

Figure 10: 2D slider bearing model. thus,

We also have $p_{1}^{\prime}=p_{4}^{\prime}=p_{\mathrm{atm}}$ and $Q_{\mathrm{AB}}^{\prime}=Q_{\mathrm{BC}}^{\prime}=Q_{\mathrm{CD}}^{\prime}$;

$$
\begin{aligned}
& p_{2}^{\prime}=p_{\mathrm{atm}}-\frac{6 \mu U_{s} a b}{(a+b+c) h_{0}^{2}}, \\
& p_{3}^{\prime}=p_{\mathrm{atm}}+\frac{6 \mu U_{s} b c}{(a+b+c) h_{0}^{2}} .
\end{aligned}
$$

Under the condition that there are the same pressure distributions for these two cases with a dimple and with a slip region, respectively, that is, $p_{2}=p_{2}^{\prime}$ and $p_{3}=p_{3}^{\prime}$, and by combining (14) and (16) or (13) and (17), we thus obtain

$$
U_{s}=\frac{(a+b+c)\left(h_{d}-h_{0}\right) h_{0}{ }^{2}}{(a+c) h_{d}{ }^{3}+b h_{0}{ }^{3}} U_{0} .
$$

This expression (18) indicates that the slip velocity has a relation to the geometry parameters of surface texture and the movement condition. For a given model with determinate surface texture and movement condition, the corresponding slip velocity induced by surface texture is also determinate and its value is one and only value.

\section{Conclusions}

Comparative analyses about the influences of surface texture and boundary slip on the tribological performances of slider bearings are conducted. The analysis results indicate that the tribological characteristics of slip and textured surfaces have strong similarity. For a given texture configuration, there is one and only slip velocity to make their tribological performances equivalent. A corresponding relation between slip velocity and texture parameters is also deduced based on 2D slider bearing with one dimple texture. The size of slip velocity is directly related to the texture geometry parameters including its distribution position. These results in this work are useful to further understand the slip phenomenon as well as the relationship between boundary slip and surface texture.

\section{Nomenclature}

$u / v / w$ : Velocity components

$p: \quad$ Pressure

$P: \quad$ Dimensionless pressure

$\rho: \quad$ Density

$\mu: \quad$ Viscosity

$r_{i}: \quad$ Inner radius

$r_{o}: \quad$ Outer radius

$r: \quad$ Radius

$l_{d}$ : Dimple length

$l_{c}$ : Dimple cell length

$U_{s}$ : Slip velocity

$b$ : $\quad$ Slip length

$U_{0}$ : Moving velocity

$U_{i}$ : Inner-fluid velocity

$f$ : $\quad$ Slip-intensity factor

$n: \quad$ Surface normal vector

$\omega$ : Rotational speed

$p_{\text {atm }}$ Ambient pressure

$x / y / z$ : Cartesian coordinates 


$\begin{array}{ll}\theta_{t}: & \text { Circumferential angle of dimple region } \\ \theta_{p}: & \text { Circumferential angle of bearing pad } \\ B_{t}: & \text { Radial width of dimple region } \\ B_{p}: & \text { Radial width of bearing pad } \\ h / h_{c} / h_{d} / h_{0}: & \text { Fluid film thickness } \\ \tau_{c}: & \text { Critical shear stress } \\ \tau_{0}: & \text { Wall shear stress } \\ \tau_{s}: & \text { Shear stress of slip surface } \\ u_{s} / v_{s} / w_{s}: & \text { Velocity components of } U_{s} \\ u_{i} / v_{i} / w_{i}: & \text { Velocity components of } U_{i} \\ x_{n} / y_{n} / z_{n}: & \text { Cartesian coordinates where } n=-z_{n} \\ L: & \text { Circumferential length of bearing pad } \\ W: & \text { Load-carrying capacity } \\ a / b / c: & \text { Length of different bearing regions } \\ \mathrm{A} / \mathrm{B} / \mathrm{C} / \mathrm{D}: & \text { Position marks } \\ \mathrm{Q}: & \text { Flow rate. }\end{array}$

\section{Superscript/Subscript}

': $\quad$ Slip bearing

slip: Slip-surface zone

noslip: Noslip-surface zone

1/2/3/4: The position A/B/C/D.

\section{Conflict of Interests}

The authors declare that there is no conflict of interests regarding the publication of this paper.

\section{Acknowledgments}

This work is financially supported by the National Natural Science Foundation of China (no. 51135004) and the Teacher Research Support Program of Xi'an Jiaotong University (no. DW013217K0000001).

\section{References}

[1] M. Arghir, N. Roucou, M. Helene, and I. Frene, “Theoretical analysis of the incompressible laminar flow in a macro-roughness cell," Journal of Tribology, vol. 125, no. 2, pp. 309-318, 2003.

[2] R. Salant and A. Fortier, "Numerical analysis of a slider bearing with a heterogeneous slip/no-slip surface," Tribology Transactions, vol. 47, no. 3, pp. 328-334, 2004.

[3] M. Mahmoud and S. Waheed, "Effects of slip and heat generation/absorption on MHD mixed convection flow of a micropolar fluid over a heated stretching surface," Mathematical Problems in Engineering, vol. 2010, Article ID 579162, 20 pages, 2010.

[4] Q. Lin, Z. Wei, and N. Wang, "Optimum design of recess parameters for a high-speed hybrid journal bearing using fluidstructure interaction and improved orthogonal experiment method," Journal of the Balkan Tribological Association, vol. 21, no. 2, pp. 300-313, 2015.

[5] M. Fesanghary and M. M. Khonsari, “Topological and shape optimization of thrust bearings for enhanced load-carrying capacity," Tribology International, vol. 53, pp. 12-21, 2012.

[6] D. Lu, W. Zhao, B. Lu, and J. Zhang, "Cage speed of hydrodynamic rolling hybrid bearings," Tribology Letters, vol. 51, no. 3, pp. 303-309, 2013.
[7] M. E. Salama, "The effect of macro-roughness on the performance of parallel thrust bearings," Proceedings of the Institution of Mechanical Engineers, vol. 163, no. 1, pp. 149-161, 1950.

[8] D. B. Hamilton, J. A. Walowit, and C. M. Allen, "A theory of lubrication by microirregularities," Journal of Basic Engineering, vol. 88, no. 1, pp. 177-185, 1966.

[9] J. N. Anno, J. A. Walowit, and C. M. Allen, "Microasperity lubrication," Journal of Lubrication Technology, vol. 90, no. 2, pp. 351-355, 1968.

[10] K. Tønder, "Inlet roughness tribodevices: dynamic coefficients and leakage," Tribology International, vol. 34, no. 12, pp. 847$852,2001$.

[11] V. Brizmer, Y. Kligerman, and I. Etsion, "A laser surface textured parallel thrust bearing," Tribology Transactions, vol. 46, no. 3, pp. 397-403, 2003.

[12] A. V. Olver, M. T. Fowell, H. A. Spikes, and I. G. Pegg, “'Inlet suction, a load support mechanism in non-convergent, pocketed, hydrodynamic bearings," Proceedings of the Institution of Mechanical Engineers, Part J: Journal of Engineering Tribology, vol. 220, no. 2, pp. 105-108, 2006.

[13] A. Gherca, A. Fatu, M. Hajjam, and P. Maspeyrot, "Influence of surface geometry on the hydrodynamic performances of parallel bearings in transient flow conditions," Tribology Transactions, vol. 56, no. 6, pp. 953-967, 2013.

[14] K. Yagi and J. Sugimura, "Balancing wedge action: a contribution of textured surface to hydrodynamic pressure generation," Tribology Letters, vol. 50, no. 3, pp. 349-364, 2013.

[15] M. Fowell, A. V. Olver, A. D. Gosman, H. A. Spikes, and I. Pegg, "Entrainment and inlet suction: two mechanisms of hydrodynamic lubrication in textured bearings," Journal of Tribology, vol. 129, no. 2, pp. 336-347, 2007.

[16] M. B. Dobrica, M. Fillon, M. D. Pascovici, and T. Cicone, "Optimizing surface texture for hydrodynamic lubricated contacts using a mass-conserving numerical approach," Proceedings of the Institution of Mechanical Engineers, Part J: Journal of Engineering Tribology, vol. 224, no. 8, pp. 737-750, 2010.

[17] J. Ji, Y. Fu, and Q. Bi, "The influence of partially textured slider with orientation ellipse dimples on the behavior of hydrodynamic lubrication," Industrial Lubrication and Tribology, vol. 66, no. 2, pp. 161-167, 2014.

[18] M. Qiu, B. R. Minson, and B. Raeymaekers, "The effect of texture shape on the friction coefficient and stiffness of gaslubricated parallel slider bearings," Tribology International, vol. 67, pp. 278-288, 2013.

[19] L. Hao, Y. Meng, and C. Chen, "Experimental investigation on effects of surface texturing on lubrication of initial line contacts," Lubrication Science, vol. 26, no. 5, pp. 363-373, 2014.

[20] J. P. Rothstein, "Slip on superhydrophobic surfaces," Annual Review of Fluid Mechanics, vol. 42, pp. 89-109, 2010.

[21] B. Bhushan, Y. Wang, and A. Maali, "Boundary slip study on hydrophilic, hydrophobic, and superhydrophobic surfaces with dynamic atomic force microscopy," Langmuir, vol. 25, no. 14, pp. 8117-8121, 2009.

[22] F. Feuillebois, M. Z. Bazant, and O. I. Vinogradova, "Effective slip over superhydrophobic surfaces in thin channels," Physical Review Letters, vol. 102, no. 2, Article ID 026001, 2009.

[23] B. Ono and Y. Yamamoto, "Possibility of slip in hydrodynamic oil films under sliding contact conditions," Lubrication Science, vol. 14, no. 3, pp. 303-320, 2002.

[24] K. Bhattacharyya, G. C. Layek, and R. S. R. Gorla, "Slip effect on boundary layer flow on a moving flat plate in a parallel free 
stream," International Journal of Fluid Mechanics Research, vol. 39, no. 5, pp. 438-447, 2012.

[25] Y. Zhang, "Review of hydrodynamic lubrication with interfacial slippage," Journal of the Balkan Tribological Association, vol. 20, no. 4, pp. 522-538, 2014.

[26] H. A. Spikes, "The half-wetted bearing. Part 1: extended Reynolds equation," Proceedings of the Institution of Mechanical Engineers, Part J: Journal of Engineering Tribology, vol. 217, no. 1, pp. 1-14, 2003.

[27] A. E. Fortier and R. F. Salant, "Numerical analysis of a journal bearing with a heterogeneous slip/no-slip surface," Journal of Tribology, vol. 127, no. 4, pp. 820-825, 2005.

[28] C.-K. Chen, H.-Y. Lai, and W.-F. Chen, "Unsteady unidirectional flow of second-grade fluid through a microtube with wall slip and different given volume flow rate," Mathematical Problems in Engineering, vol. 2010, Article ID 416837, 17 pages, 2010.

[29] C. Wu, "Performance of hydrodynamic lubrication journal bearing with a slippage surface," Industrial Lubrication and Tribology, vol. 60, no. 6, pp. 293-298, 2008.

[30] Q. Lin, Z. Wei, N. Wang, and W. Chen, "Effect of large-area texture/slip surface on journal bearing considering cavitation," Industrial Lubrication and Tribology, vol. 67, no. 3, pp. 216-226, 2015.

[31] Q. Lin, Z. Wei, N. Wang, and W. Chen, "Effects of large-area textured/slip surface on slider bearing," Journal of the Balkan Tribological Association, vol. 21, no. 1, pp. 12-23, 2015.

[32] F. Aurelian, M. Patrick, and H. Mohamed, "Wall slip effects in (elasto) hydrodynamic journal bearings," Tribology International, vol. 44, no. 7-8, pp. 868-877, 2011.

[33] R. Sharma, A. Ishak, and I. Pop, "Partial slip flow and heat transfer over a stretching sheet in a nanofluid," Mathematical Problems in Engineering, vol. 2013, Article ID 724547, 7 pages, 2013.

[34] V. G. Marian, M. Kilian, and W. Scholz, "Theoretical and experimental analysis of a partially textured thrust bearing with square dimples," Proceedings of the Institution of Mechanical Engineers, Part J: Journal of Engineering Tribology, vol. 221, no. 7, pp. 771-778, 2007. 


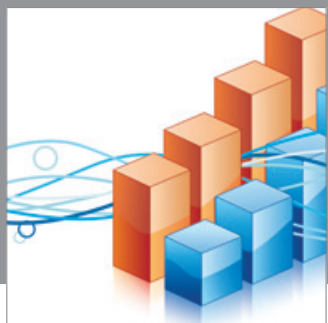

Advances in

Operations Research

mansans

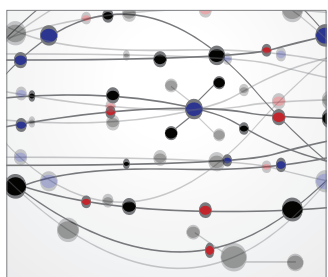

The Scientific World Journal
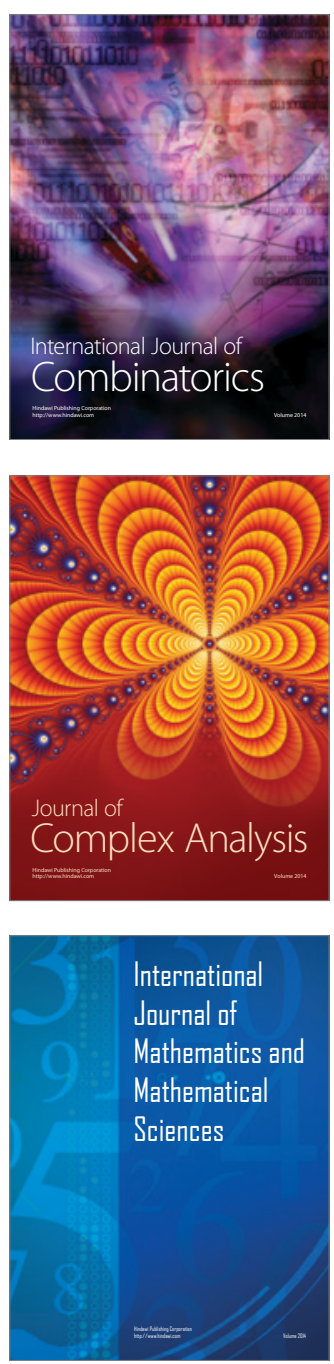
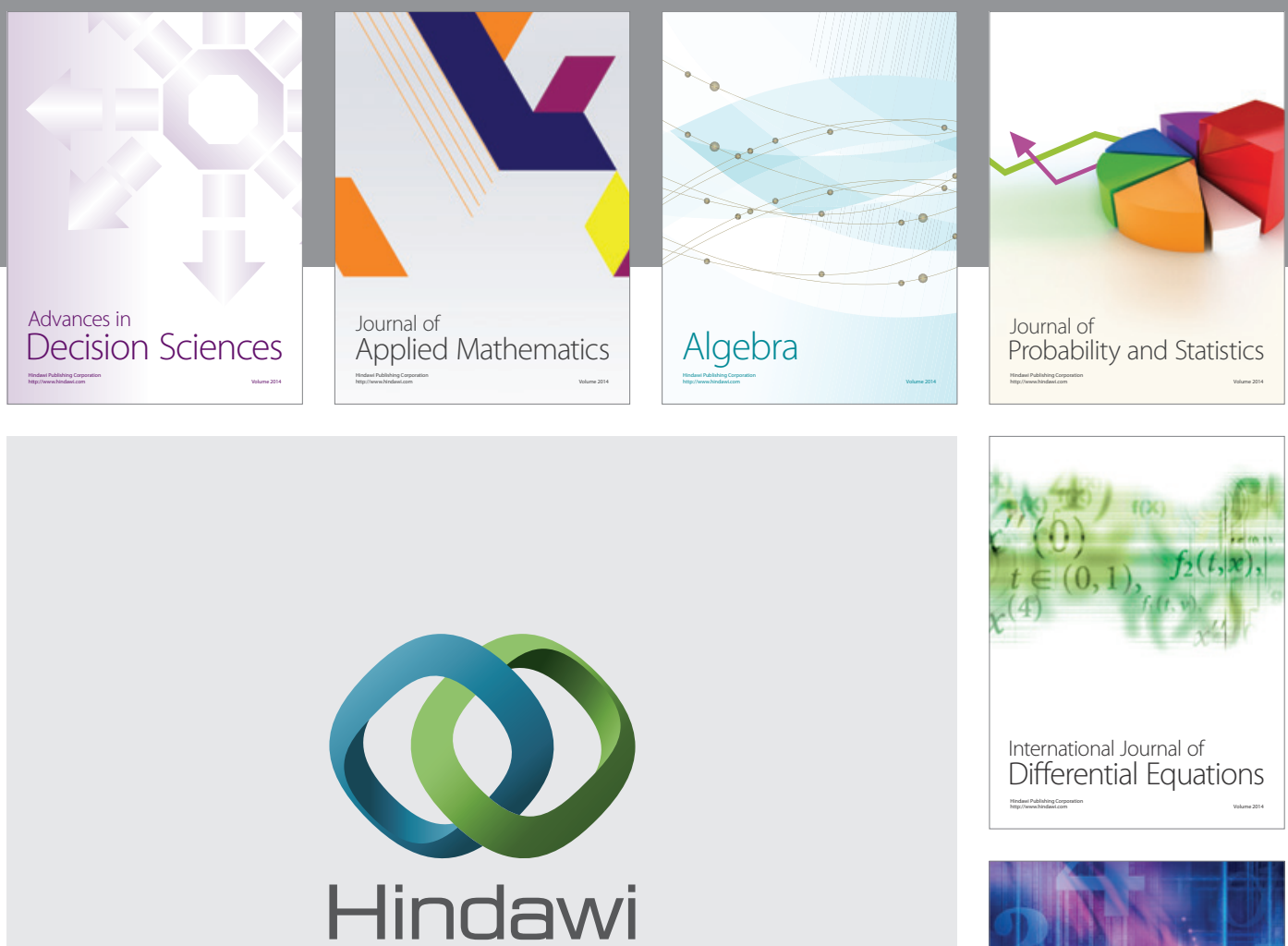

Submit your manuscripts at http://www.hindawi.com
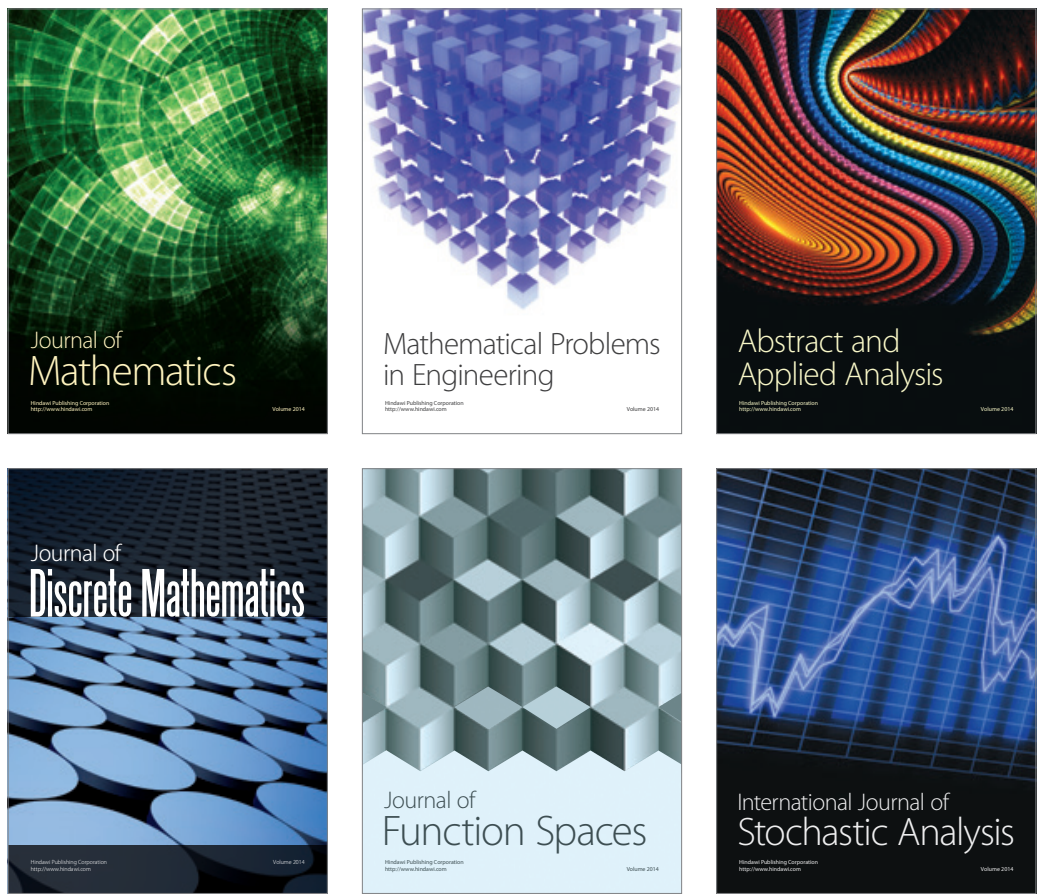

Journal of

Function Spaces

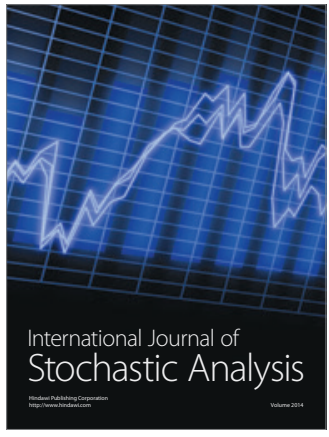

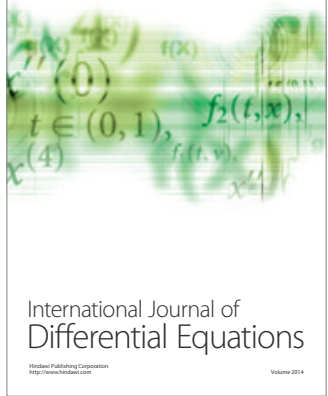
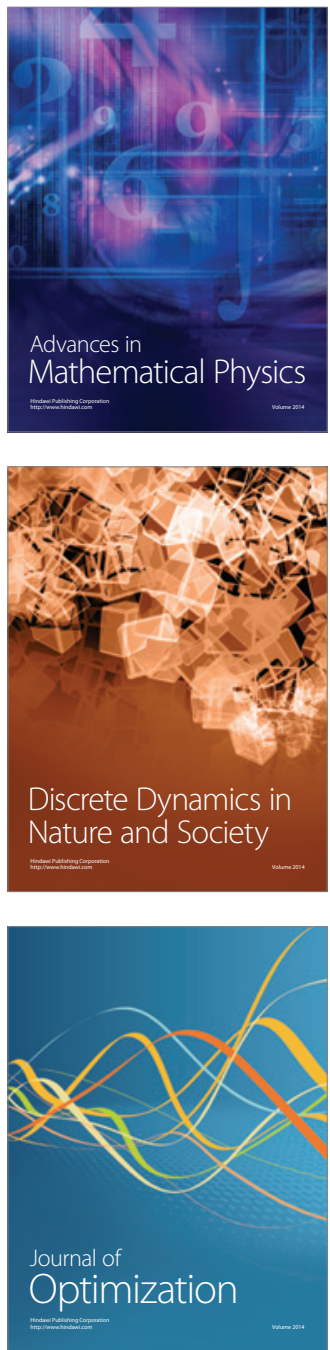